\title{
Floating millivolt reference for PTAT current generation in Subthreshold MOS LSIs
}

\author{
Ken Ueno, Tetsuya Hirose, Tetsuya Asai, and Yoshihito Amemiya \\ Department of Electrical Engineering \\ Hokkaido University, Sapporo 060-0814 Japan \\ Tel: +81-11-706-6080, Fax: +81-11-706-7890 \\ E-mail: k_ueno@sapiens-ei.eng.hokudai.ac.jp
}

\begin{abstract}
A floating millivolt reference circuit to generate a PTAT current was developed by using MOSFETs operated in the subthreshold region. The circuit generates a floating voltage of about $10 \mathrm{mV}$. The variations in the reference are $\pm 2.7 \%$ in a temperature range from -20 to $100{ }^{\circ} \mathrm{C}$. The accuracy of the reference circuit can be improved to $\pm 0.3 \%$ with a correction technique using a curvature-correction circuit. The total power consumption of the circuit was $4.6 \mu \mathrm{W}$ at $100{ }^{\circ} \mathrm{C}$.
\end{abstract}

\section{INTRODUCTION}

Intelligent-network systems with various smart-sensor devices are expected to be developed that will spread all over the world to enable infrastructures to be constructed for the information age. Such network systems will require a huge number of sensor LSIs that measure various physical data from our surroundings, store and process these measured data, and output them on demand. As these sensors must operate for long periods of time, the available energy resources -whether small batteries, energy harvesting, or both- limit their overall operation. One possible method of minimizing the energy they consume is to design a circuit in the subthreshold region of MOSFETs.

The sensing of temperature by these LSIs is one of their most fundamental applications. A sensory signal that is proportional to absolute temperature (PTAT) is necessary to construct a temperature sensor. However, as the subthreshold current of MOSFET is an exponential function of the gate-source voltage, the PTAT current cannot be generated easily. In this paper, we propose a floating millivolt reference circuit that can be used to generate PTAT current in circuits [1]. In the following, Section II describes the method of generating PTAT current for the temperature sensor, Section III presents the floating millivolt reference with the results of SPICE simulation, and Section IV describes the curvature-correction techniques to obtain a high-accuracy reference.

\section{Generation of PTAT CURREnt}

The subthreshold current $I_{D}$ of a MOSFET is an exponential function of gate-source voltage $V_{G S}$ and is given by

$$
I_{D}=I_{0} \exp \left(\frac{V_{G S}-V_{T H}}{\eta V_{T}}\right),
$$

where $I_{0}$ is a process-dependent parameter, $\eta$ is the subthreshold slope factor, and $V_{T}\left(=k_{B} T / e\right)$ is the thermal voltage [2].
Assume that two MOSFETs are biased with two different voltages $V_{G S 1}$ and $V_{G S 2}$ to generate two subthreshold currents $I_{D 1}$ and $I_{D 2}$. The difference in the current ratios is given by

$$
\frac{I_{D 2}}{I_{D 1}}-\frac{I_{D 1}}{I_{D 2}}=\exp \left(\frac{V_{G S 2}-V_{G S 1}}{\eta V_{T}}\right)-\exp \left(-\frac{V_{G S 2}-V_{G S 1}}{\eta V_{T}}\right) \text {. }
$$

Equation (2) can be rewritten for a voltage difference of $\left|V_{G S 2}-V_{G S 1}\right|<\eta V_{T}$ as

$$
\frac{I_{D 2}}{I_{D 1}}-\frac{I_{D 1}}{I_{D 2}}=2 \frac{V_{G S 2}-V_{G S 1}}{\eta V_{T}}
$$

The reciprocal of Eq. (3) is expressed as

$$
\frac{1}{\frac{I_{D 2}}{I_{D 1}}-\frac{I_{D 1}}{I_{D 2}}}=\frac{\eta V_{T}}{2\left(V_{G S 2}-V_{G S 1}\right)}=\frac{\eta k_{B}}{2 e\left(V_{G S 2}-V_{G S 1}\right)} T .
$$

The PTAT characteristic can be obtained for a fixed temperature-independent voltage difference of $\left|V_{G S 2}-V_{G S 1}\right|$.

In practical circuit implementation, the division and the subtraction of the currents can be performed by translinear circuit and current mirror circuit [1]. However, generating a small temperature-independent floating reference voltage $(\sim$ $10 \mathrm{mV}$ ) to generate the PTAT signal in Eq. (4) is not easy using a MOSFET operated in the subthreshold region. In the following sections, we develop a floating millivolt reference circuit.

\section{Flohting Reference Circuit}

\section{A. Circuit Configuration}

A bandgap reference circuits are widely used to obtain a constant reference voltage and a floating reference voltage [3]. However, they are not suitable for our purpose because they need large resistors with high resistance for low-current operation in the subthreshold region. Buck and others proposed a new CMOS bandgap reference circuit without the use of resistors [4]. We modified their circuit to operate in the subthreshold region and to generate a floating millivolt reference.

Figure 1 shows the circuit configuration. The circuit consists of two diode-connected transistors $\left(\mathrm{M}_{\mathrm{D} 1}, \mathrm{M}_{\mathrm{D} 2}\right)$ and two differential pairs $\left(\mathrm{M}_{1}-\mathrm{M}_{2}, \mathrm{M}_{3}-\mathrm{M}_{4}\right)$. We set the aspect ratios $K$ of these transistors such that

$$
K_{D 1}>K_{D 2}, K_{1}<K_{2} \text {, and } K_{3}>K_{4} .
$$




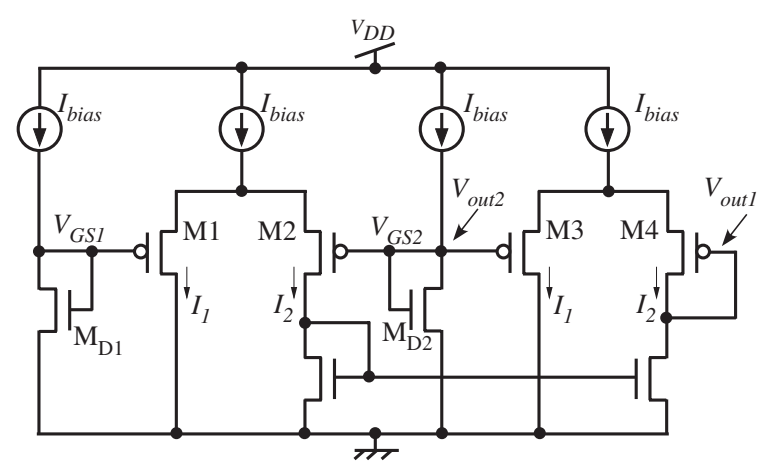

Fig. 1. Floating voltage reference circuit.

The circuit generates output voltages on output terminals $\left(V_{\text {out } 1}, V_{\text {out } 2}\right)$, and the difference in the voltages is the floating millivolt reference voltage. The floating reference voltage is equal to the sum of the differences in threshold voltage for different transistor size. The threshold voltage of a transistor depends on the length and width of its channels [5]. Therefore, the difference in threshold voltage can be used to generate a floating millivolt reference voltage. Output voltages $V_{\text {out } 1}$ and $V_{\text {out } 2}$ have a negative dependence on temperature, whereas the difference in the voltage of $V_{\text {out } 2}-V_{\text {out } 1}$ shows a temperatureinsensitive constant reference voltage. The details on the circuit operation are as follows.

The diode-connected MOSFETs $\mathrm{M}_{\mathrm{D} 1}$ and $\mathrm{M}_{\mathrm{D} 2}$ are operated in the subthreshold region, and their gate-source voltages $V_{G S, D 1}$ and $V_{G S, D 2}$ are given by

$$
V_{G S, D i}=V_{T H, D i}+\eta V_{T} \ln \left(\frac{I_{\text {bias }}}{K_{D i} I_{0}}\right) \quad(i=1,2) .
$$

The difference in the gate-source voltages $\Delta V_{G S, D}$ in $\mathrm{M}_{\mathrm{D} 1}$ and $\mathrm{M}_{\mathrm{D} 2}$ can be expressed as

$$
\Delta V_{G S, D}=\Delta V_{T H, D 21}+\eta V_{T} \ln \left(\frac{K_{D 1}}{K_{D 2}}\right),
$$

where $\Delta V_{T H, D 21}\left(=V_{T H, D 2}-V_{T H, D 1}\right)$ is the difference in the threshold voltages. The resulting currents $I_{1}$ and $I_{2}$ in differential pair $\mathrm{M}_{1}-\mathrm{M}_{2}$ are given by

$$
I_{i}=K_{i} I_{0} \exp \left(\frac{V_{s}-V_{G S, D i}-V_{T H i}}{\eta V_{T}}\right)(i=1,2),
$$

where $V_{s}$ is the common-source node voltage of the differential pair. Current $I_{2}$ is copied into the $\mathrm{M}_{4}$ of the differential pair $\mathrm{M}_{3}-\mathrm{M}_{4}$. Because the currents in transistors $\mathrm{M}_{3}$ and $\mathrm{M}_{4}$ are $I_{1}$ and $I_{2}$, gate-source voltages $V_{G S, 3}$ and $V_{G S, 4}$ are given by

$$
V_{G S, i+2}=V_{T H, i+2}+\eta V_{T} \ln \left(\frac{I_{i}}{K_{i+2} I_{0}}\right) \quad(i=1,2) .
$$

The difference $\Delta V_{R E F}\left(=V_{\text {out } 2}-V_{\text {out } 1}\right)$ in the output voltages is given by

$$
\Delta V_{R E F}=V_{G S, 4}-V_{G S, 3} .
$$

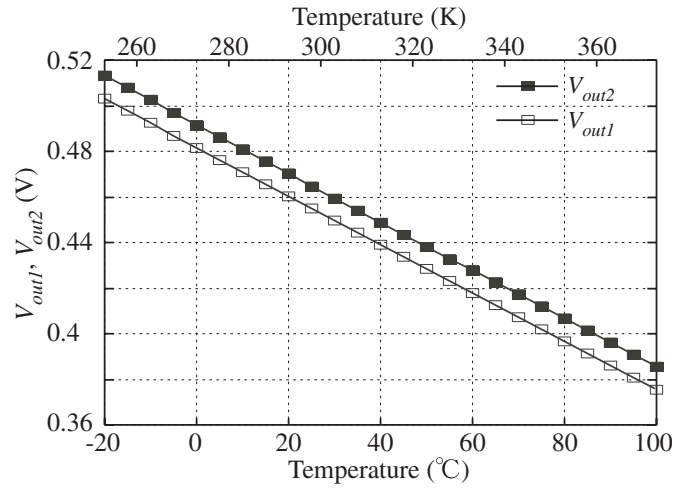

Fig. 2. Simulated output voltages $V_{\text {out } 1}$ and $V_{\text {out } 2}$.

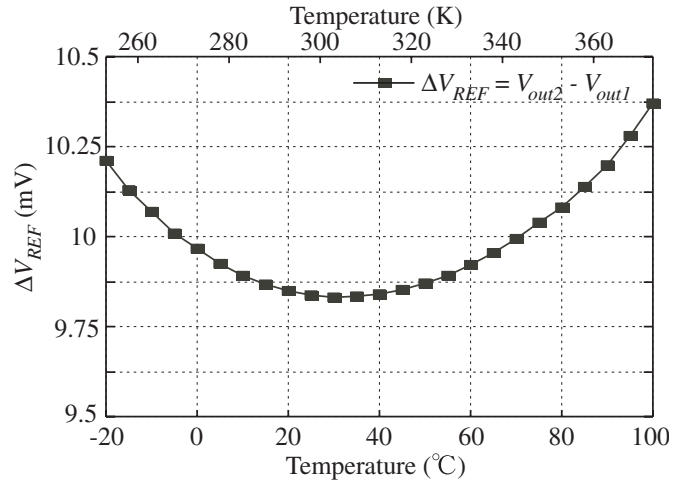

Fig. 3. Floating reference voltage $\Delta V_{R E F}$.

Equation (10) can be rewritten from Eqs. (7), (8), and (9) as

$$
\begin{aligned}
\Delta V_{R E F}= & \Delta V_{T H, 43}+\Delta V_{T H, 12}-\Delta V_{T H, D 21} \\
& +\eta V_{T} \ln \left(\frac{K_{3} K_{2} K_{D 2}}{K_{4} K_{1} K_{D 1}}\right)
\end{aligned}
$$

where $\Delta V_{T H, 43}\left(=V_{T H, 4}-V_{T H, 3}\right)$ and $\Delta V_{T H, 12}\left(=V_{T H, 1}-\right.$ $\left.V_{T H, 2}\right)$ are the differences in the threshold voltages. We set the aspect ratios $K$, with condition of Eq.(5), such that

$$
K_{3} K_{2} K_{D 2}=K_{4} K_{1} K_{D 1} .
$$

Then, the difference in voltage is constant with temperature. The floating voltage reference of the circuit is given by

$$
\Delta V_{R E F}=\Delta V_{T H, 43}+\Delta V_{T H, 12}-\Delta V_{T H, D 21} .
$$

We can obtain the floating millivolt reference by adjusting the size of the transistors.

\section{B. Simulation Results}

We confirmed the operation of the circuit by SPICE simulation, assuming a set of $0.35-\mu \mathrm{m} 2 \mathrm{P} 4 \mathrm{M}$-standard CMOS parameters and a $1.5-\mathrm{V}$ power supply.

Figure 2 shows simulated output voltages of $V_{\text {out } 1}$ and $V_{\text {out } 2}$ as a function of temperature from -20 to $100{ }^{\circ} \mathrm{C}$. Both the output voltages decrease linearly with temperature and maintain a constant difference in voltage. Figure 3 shows the floating reference voltage $\Delta V_{R E F}$. The difference is 
almost constant and was about $10 \mathrm{mV}$. However, the floating reference voltage increases at low- and high-temperatures, and has nonlinear characteristics due to nonlinearity of the diode-connected transistors. The variations in the reference voltage were about $\pm 2.7 \%$. This nonlinearity degenerates the precision with which the PTAT current is generated.

\section{Curvature-Correction Techniques}

We develop curvature-correction techniques to improve the accuracy of the floating reference voltage. Several curvaturecorrection techniques have been presented in the literatures [6]-[9]. We use an idea based on the piecewise-linear curvature-correction technique [9]. The details on circuit operation are as follows.

\section{A. Curvature-Correction Architecture}

The correction is performed by changing the bias current in diode-connected transistor $\mathrm{M}_{\mathrm{D} 2}$. The bias current in diodeconnected transistor $\mathrm{M}_{\mathrm{D} 2}$ is increased at low- and hightemperatures by using curvature-correcting current $I_{\text {curv }}$.

The gate-source voltage $V_{G S, D 2}$ of transistor $\mathrm{M}_{\mathrm{D} 2}$ is now given by

$$
V_{G S, D 2}=V_{T H, D 2}+\eta V_{T} \ln \left(\frac{I_{b i a s}+I_{c u r v}}{K_{D 2} I_{0}}\right) .
$$

The difference $\Delta V_{G S, D}$ in the gate-source voltages in $\mathrm{M}_{\mathrm{D} 1}$ and $\mathrm{M}_{\mathrm{D} 2}$ can be expressed as

$$
\Delta V_{G S, D}=\Delta V_{T H, D 21}+\eta V_{T} \ln \left(\frac{K_{D 1}\left(I_{\text {bias }}+I_{c u r v}\right)}{K_{D 2} I_{\text {bias }}}\right) \text {. }
$$

The difference in the output voltage $\Delta V_{R E F}$ can be given by

$$
\begin{aligned}
\Delta V_{R E F}= & \Delta V_{T H, 43}+\Delta V_{T H, 12}-\Delta V_{T H, D 21} \\
& +\eta V_{T} \ln \left(\frac{I_{\text {bias }}}{I_{\text {bias }}+I_{\text {curv }}}\right) .
\end{aligned}
$$

Therefore, the curvature-correcting current $I_{\text {curv }}$ suppress the increase in the output floating reference at low- and hightemperatures.

\section{B. Circuit for Generating Curvature-Correcting Current}

The curvature-correction circuit we developed uses two curvature-correcting currents $I_{\text {curv } 1}$ and $I_{\text {curv } 2}$. We apply the current $I_{\text {curv }}$ to node $V_{\text {out } 2}$ such that

$$
I_{\text {curv }}= \begin{cases}I_{\text {curv } 1} & \text { (at low temp.) } \\ 0 & \text { (at middle temp.) } \\ I_{\text {curv } 2} & \text { (at high temp.). }\end{cases}
$$

The architecture to generate the curvature-correcting current is shown in Fig. 4. We use a PTAT current $I_{P T A T}$ and temperature independent currents $I_{R E F 1}, I_{R E F 2}$ to generate the curvature-correcting current. The architecture is based on the nodal subtraction of PTAT current $I_{P T A T}$ and the reference currents of $I_{R E F 1}$ and $I_{R E F 2}$. The reference currents and PTAT current are set so that $I_{R E F 1}<I_{R E F 2}$ and that $I_{P T A T}$ has intersecting points with both the reference currents at different temperatures $T_{1}$ and $T_{2}$ as shown in Fig. 4(a). At
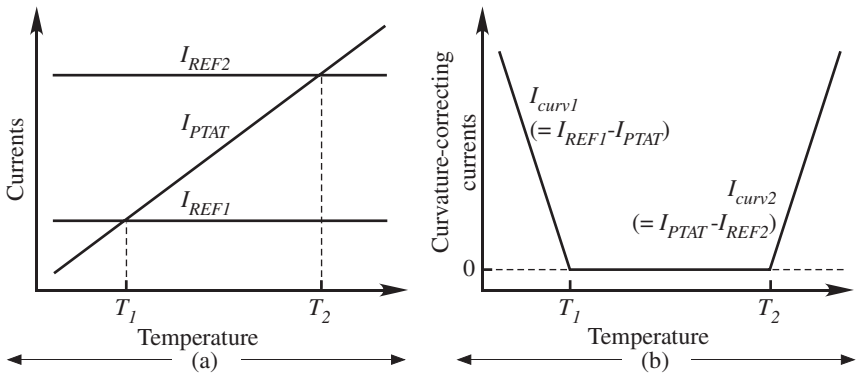

Fig. 4. Architecture for generating curvature-correcting current ( $I_{\text {curv } 1}$, $I_{\text {curv2 } 2}$ ) generation.

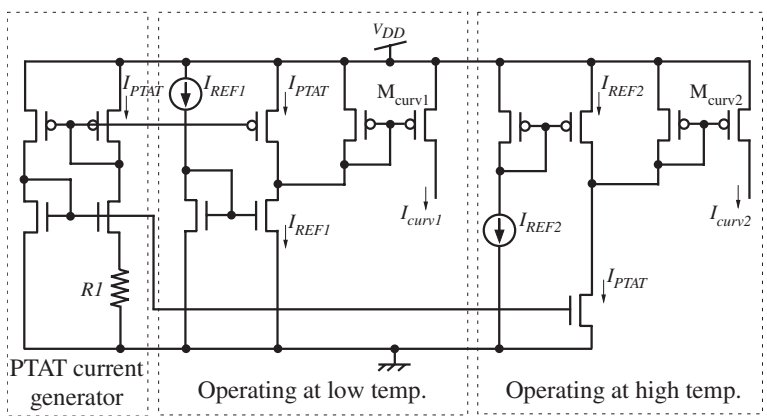

Fig. 5. Circuit for generating curvature-correcting current.

temperatures lower than $T_{1}, I_{R E F 1}-I_{P T A T}$ decreases with temperature. At temperatures higher than $T_{2}, I_{P T A T}-I_{R E F 2}$ increases with temperature. Figure. 4(b) shows their subtraction currents $I_{\text {curv } 1}, I_{\text {curv } 2}$. From this way, the curvaturecorrecting current $I_{\text {curv }}$ is generated and applied to node $V_{\text {out } 2}$. The curvature-correcting current can be summarized as

$$
I_{\text {curv }}= \begin{cases}I_{R E F 1}-I_{P T A T} & \text { (at low temp.) } \\ 0 & \text { (at middle temp.) } \\ I_{P T A T}-I_{R E F 2} & \text { (at high temp.). }\end{cases}
$$

Figure 5 shows the circuit we propose for generating curvature-correcting current, which consists of a PTAT current generator, and two subcircuits that generate curvaturecorrecting current: one works at low temperature and the other at high temperature.

The PTAT current for the lower temperature range is less than the reference currents: $I_{P T A T}<I_{R E F 1}<I_{R E F 2}$, and, therefore, the output transistor $\mathrm{M}_{\text {curv1 }}$ generates subtracted current $I_{R E F 1}-I_{P T A T}$. The output transistor $\mathrm{M}_{\text {curv2 }}$ does not generate any current because transistor $\mathrm{M}_{\mathrm{curv} 2}$ is in the off state. The PTAT current for a middle temperature range increases more than reference current $I_{R E F 1}$ and is less than reference current $I_{R E F 2}: I_{R E F 1}<I_{P T A T}<I_{R E F 2}$. The output transistors $\mathrm{M}_{\text {curv1 }}$ and $\mathrm{M}_{\text {curv2 }}$ do not generate any currents. The PTAT current for a higher temperature range increases more than the reference currents: $I_{R E F 1}<I_{R E F 2}<$ $I_{P T A T}$, and, therefore, the output transistor $\mathrm{M}_{\mathrm{curv} 2}$ generates subtracted current $I_{P T A T}-I_{R E F 2}$. 


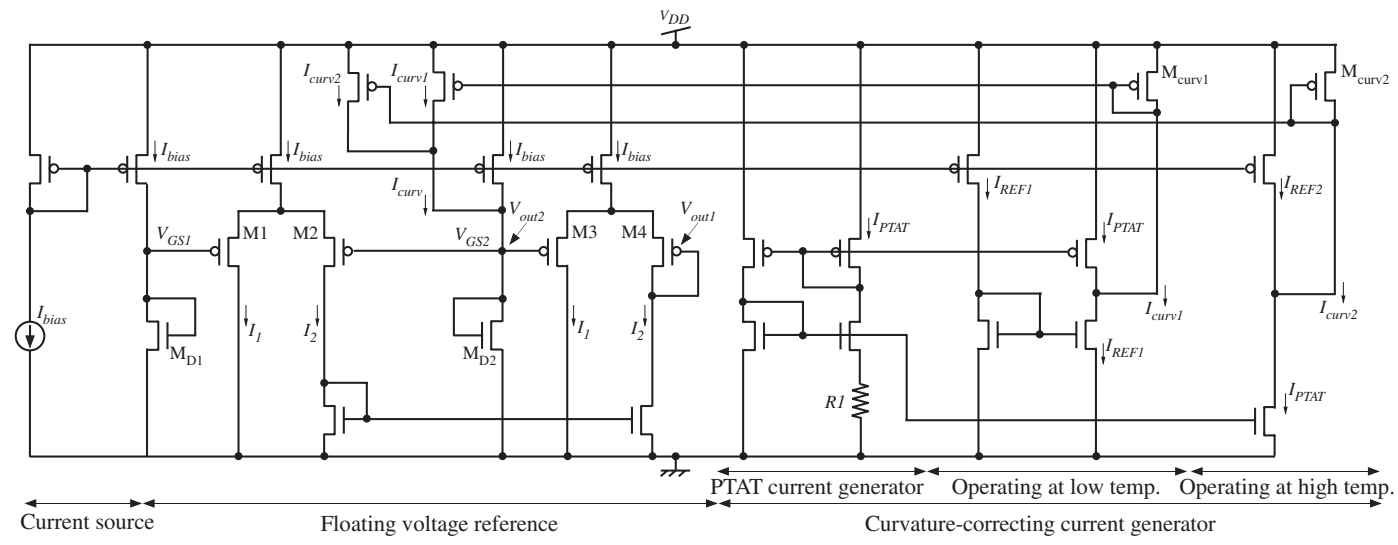

Fig. 6. The floating reference circuit including curvature correction circuit.

\section{Simulation Results}

We demonstrated the curvature-correction technique by SPICE simulation. Figure 6 shows the entire circuit. The bias current of $I_{\text {bias }}$ was set to $100 \mathrm{nA}$. The reference currents $I_{R E F 1}$ and $I_{R E F 2}$ in the curvature-correction circuit were set to $160 \mathrm{nA}$ and $190 \mathrm{nA}$ by changing the current gain factors of the current mirror. Figure 7 shows the simulated curvature-correcting current as a function of temperature. The correcting current $I_{\text {curv } 1}$ was generated at lower temperature and decreased with temperature. The correcting current $I_{\text {curv } 2}$ was generated at higher temperature and increased with temperature. These correcting currents were applied to node $V_{\text {out } 2}$ through the current mirror. Figure 8 shows the output floating reference voltage. The uncorrected reference voltage is also plotted. The variations in the reference voltage were reduced to $\pm 0.3 \%$ from uncorrected reference variations of $\pm 2.7 \%$. The maximum power consumption of the circuit was $4.6 \mu \mathrm{W}$ at $100{ }^{\circ} \mathrm{C}$. The floating reference voltage circuit requires a reference current, and the reference circuit in [10] can be used for our purpose.

\section{Conclusion}

We developed a floating millivolt reference circuit to generate PTAT current. The circuit operation was confirmed by simulation with $0.35-\mu \mathrm{m}$ standard CMOS parameters. The reference voltage was about $10 \mathrm{mV}$, and the variations were $\pm 2.7 \%$ in a temperature range from -20 to $100{ }^{\circ} \mathrm{C}$. The curvature-correction circuit was also developed to improve the accuracy of the reference voltage. The accuracy of the reference circuit could be improved to $\pm 0.3 \%$ by using this correction technique. The total power consumption of the circuit was $4.6 \mu \mathrm{W}$ at $100{ }^{\circ} \mathrm{C}$.

\section{REFERENCES}

[1] K Ueno, T Hirose, T Asai, Y Amemiya, "Ultralow-power smart temperature sensor with subthreshold CMOS circuits," Proc. International Symposium on Intelligent Signal Processing and Communication Systems, pp. 546-549, Dec. 2006.

[2] Y. Taur, T.H. Ning, "Fundamentals of Modern VLSI Devices," Cambridge University Press, 2002.

[3] P.E. Allen, D.R. Holberg, "CMOS analog circuit design second edition," New York Oxford University Press, 2002.

[4] A.E. Buck, C.L McDonald, S.H Lewis, T.R Viswanathan, "A CMOS bandgap reference without resistors," IEEE J. Solid-State Circuits, vol.37, no.1, pp.81-83, Jan. 2002.

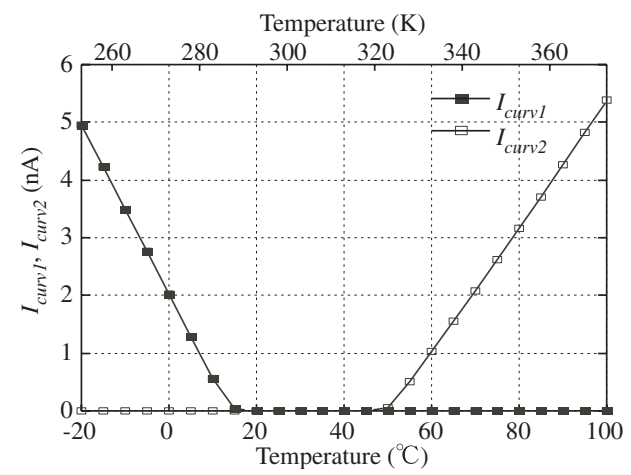

Fig. 7. The simulated curvature-correcting current.

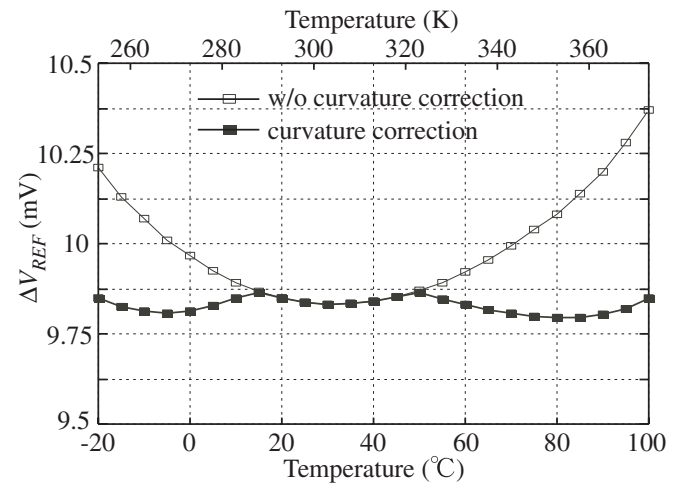

Fig. 8. The output floating reference voltages with and without the curvature correction circuit.

[5] M.C. Hsu, B.J. Sheu, "Inverse-geometry dependence of MOS transistor electrical parameters," IEEE Trans. Computer-Aided Design, vol. CAD-6, pp582-585, July. 1987.

[6] B-S. Song, P.R. Gray, "A precision curvature-compensated CMOS bandgap reference," IEEE J. Solid-State Circuits, vol. SC-18, pp. 634643, Dec. 1983.

[7] G.C.M. Meijer, P.C. Schmale, K.V. Zalinge, "A new curvature-corrected bandgap reference," IEEE J. Solid-State Circuits, vol. SC-17, pp. 11391143, Dec. 1982.

[8] M. Pertijs, K. Makinwa, J. H. Huijsing, "A CMOS smart temperature sensor with a $3 \sigma$ inaccuracy of $\pm 0.1^{\circ} \mathrm{C}$ from $-55^{\circ} \mathrm{C}$ to $125^{\circ} \mathrm{C}$," IEEE J. Solid-State Circuits, vol. 40, no. 12, pp. 2805-2815, Dec. 2005.

[9] G.A. Rincon-Mora, P.E. Allen, "A 1.1-V current-mode and piecewiselinear curvature-corrected bandgap reference," IEEE J. Solid-State Circuits, vol. 33, pp. 1551-1554, Oct. 1998.

[10] T. Hirose, T. Matsuoka, K. Taniguchi, T. Asai, Y. Amemiya, "Ultralowpower current reference circuit with low temperature dependence," IEICE Trans. Electron., Vol.E88-C, no.6, pp.1142-1147, Nov. 2004. 